\title{
Does Body Weight Account for the Declines in Sexual Activity and Incident Health Problems in Older Adults? Commentary on Jackson et al. (2019)
}

\author{
Patrick Mullie $^{1}$ (D)
}

Received: 21 September 2019 / Revised: 3 October 2019 / Accepted: 5 October 2019 / Published online: 17 October 2019

(c) Springer Science+Business Media, LLC, part of Springer Nature 2019

Using data from the English Longitudinal Study of Ageing, Jackson et al. (2019) found an association between declines in sexual activity and increased risk of cancer, coronary heart disease, and stroke. In multivariate analyses, odds ratios were adjusted for age, partnership status, ethnicity, wealth, smoking status, alcohol intake, physical activity, and depressive symptoms.

However, we believe that the observed results in multivariate analyses were not adjusted for a possible confounder, i.e., body weight. A confounder is a variable that is associated or has a relationship with both the exposure and the outcome of interest. Elevated body weight has long been associated with a number of diseases and metabolic abnormalities, many of which have high morbidity and mortality (Pi-Sunyer, 2002). Moreover, an elevated body weight has also been associated with erectile dysfunction, which can influence sexual behavior negatively (Moon, Park, \& Kim, 2019). So, without adjustment for body weight, the apparent relationship between sexual dysfunction and health problems could be possibly due to an increased body weight. In their discussion, Jackson et al. (2019) stated that: "It is possible that this association may be explained by certain risk factors associated with both cancer and erectile dysfunction, such as obesity, a high-fat diet, smoking, or alcohol use. However, as the present analyses controlled for smoking and alcohol use, it is unlikely that these confounding variables are driving the observed association."

This is a strange statement, because Jackson et al. (2019) did not adjust for body weight. In conclusion, and without adjustment for body weight, we do not agree with the statement that the development of interventions to promote sexual health and well-being at older ages may offer considerable opportunities to reduce the burden of disease in later life.

\section{References}

Jackson, S. E., Yang, L., Koyanagi, A., Stubbs, B., Veronese, N., \& Smith, L. (2019). Declines in sexual activity and function predict incident health problems in older adults: Prospective findings from the English longitudinal study of ageing. Archives of Sexual Behavior. https://doi.org/10.1007/s10508-019-1443-4.

Moon, K. H., Park, S. Y., \& Kim, Y. W. (2019). Obesity and erectile dysfunction: From bench to clinical implication. The World Journal of Men's Health, 37, 138-147.

Pi-Sunyer, F. X. (2002). The obesity epidemic: Pathophysiology and consequences of obesity. Obesity Research, 10, 97S-104S.

Publisher's Note Springer Nature remains neutral with regard to jurisdictional claims in published maps and institutional affiliations.
Patrick Mullie

patrick.mullie@skynet.be

1 Belgian Centre for Evidence-Based Medicine, Cochrane Belgium, Kapucijnenvoer 33, 3000 Leuven, Belgium 